time, levels of coagulation factors and albumin, bicytopenia, but with normal ALT and bilirubin; only AST and GGT were minimally above the upper normal limit). Esophagogastroduodenoscopy revealed esophageal varices grade I and portal gastropathy due to portal hypertension. Kayser Fleischer ring was present. Low ceruloplasmin levels and positive penicillamine test further confirmed the suspicion for Wilson's disease which was confirmed by genetic testing that showed homozygous H1069Q mutation. Once the diagnosis was established, we gathered a multidisciplinary team which included neurologist, gastroenterologist, hematologist, cardiologist, nephrologist, rheumatologist, endocrinologist, dietitian, and psychologist. There were no signs of renal tubular damage and the heart was structurally healthy.

Penicillamine was gradually introduced, but not to the maximum dose recommended by the European Society for Paediatric Gastroenterology, Hepatology and Nutrition (ESPGHAN). One week later zinc acetate was added into the therapy. We have chosen this scheme because of the risk of hematological complications at the penicillamine full dose. Vitamin D and calcium supplementation was introduced due to reduced bone density. Other supportive therapy included a copper-free diet, high-energy oral nutritional supplement adjusted for patients with liver disease, MCT oil, and gastroprotection. Two months after initiation of therapy cupriuria is threefold increased as compared to the initial values suggesting efficacy of therapy. She has not had side effects with this combination therapy.

Although the most common presentation of Wilson's disease in childhood includes liver disease, we should be aware of its possibility to present with neurological symptoms without obvious clinical signs of liver disease, despite the existence of cirrhosis. A multidisciplinary team is required to monitor possible complications of the disease, side effects of the therapy and offer psychological support to the patient and their family.

\section{AUDIT ON FIRST PAEDIATRIC ASSESSMENT OF CHILDREN REFERRED WITH SUSPECTED EPILEPSY BEFORE AND DURING PANDEMIC}

Maymunah Khries*, Helen Estyn-Jones. Queen Elizabeth Hospital, Gateshead

10.1136/archdischild-2021-europaediatrics.394

Diagnosing epilepsy can be complex, and prone to be misdiagnosed between $5-30 \%$ of the time. It is therefore important to have specialist review early in all cases of suspected epileptic seizures to facilitate subsequent care and management, as well as to reduce parental anxiety. However, Covid-19 pandemic has added extra challenge for healthcare providers to achieve and maintain this standard of practice.

The objectives are;

1) To audit health care for children with suspected epilepsy against NICE recommendation; NICE guideline recommends all children and young people presenting with a suspected epileptic seizure to be seen by a specialist in the diagnosis and management of the epilepsies within 2 weeks of presentation.

2)To determine the effect of the pandemic on the number of referrals. 3)To look at the outcomes following first assessment for suspected epileptic seizures.
1) Identification of 2 cohorts of children presenting to outpatient service for suspected seizure between March-May 2019 and March - May 2020.

2) Retrospective case notes analysis following first paediatric assessment.

1) Number of referrals declined by more than $20 \%$ during pandemic, especially from general practitioners.

2) In 2019 cohort (pre-pandemic), 55\% of the cases were seen within 14 days of referral compared to $42 \%$ in 2020 cohort ( during pandemic).

3) More than half of the referrals were diagnosed as non epileptic events after specialist review. However, the outcome was better in 2020 cohort compared to the previous year.

1) The pandemic is likely to have contributed to the decline in number of referrals and resulted in more delays to clinic appointments due to limited clinic slots imposed by the pandemic restrictions.

2) Local measures to enhance referral pathway to ensure suspected epileptic seizure cases to be seen or assessed within 14 days as per guideline;

- Clear signpost to secretaries for clinic allocations.

- Creating a group email for epilepsy team as one of the pathways for referral. This will make correspondence easier for both ends and aides in filtering process as well as expediting clinic appointment.

- Encourage a phone triage in cases where the diagnosis of epileptic event isn't obvious.

3) Liaise with IT department to add a few prompts for filtering and checklists before providing the option of 'first seizure clinic' when electronic referral is made. This is meant to facilitate in obtaining relevant information, referral checklists prior to appointment and to ensure referrals are allocated to the right clinic.

3) This audit can be used as a feedback tool for the local healthcare providers both in term of referral outcomes and raising awareness on first seizure referral.

\section{SEVERE NEUROLOGICAL SYMPTOMS IN A 7.5-MONTH- OLD GIRL WITH MEGALOBLASTIC ANAEMIA AND METHYLMALONIC ACIDURIA - CASE REPORT}

Agnieszka Słowińska*, Patryk Domarecki. Wojewódzki Specjalistyczny Szpital Dziecięcy w Olsztynie

\subsection{6/archdischild-2021-europaediatrics.395}

The case report focuses on a 7,5-month-old girl, who was admitted to our hospistal becouse of vomiting, failure to thrive, pathological somnolence and developmental regression. The girl was exclusively breastfed and mother tried to introduce new foods many times with failure. Routine and commonly used laboratory tests showed megaloblastic anaemia and vitamin B12 deficiency. Further investigation revealed methylomalonic aciduria and elevated levels of homocysteine and lactic acid, which provides additional evidence of a functional measure of intracellular B12 levels. After starting vitamin B12 supplementation, a significant improvement in the clinical condition was observed and all symptoms gradually disappeared. Further treatment included supplementation of liposomal vitamin B12, folic acid and 